\title{
In vivo multiphoton imaging of an ovarian cancer mouse model
}

Travis W. Sawyer, Faith F. Rice, Jennifer W. Koevary, Denise C. Connolly, Kathy Q. Cai, et al.

Travis W. Sawyer, Faith F. Rice, Jennifer W. Koevary, Denise C. Connolly, Kathy Q. Cai, Jennifer K. Barton, "In vivo multiphoton imaging of an ovarian cancer mouse model," Proc. SPIE 10856, Diseases in the Breast and Reproductive System V, 1085605 (26 February 2019); doi: $10.1117 / 12.2505825$

SPIE. Event: SPIE BiOS, 2019, San Francisco, California, United States 


\title{
In vivo multiphoton imaging of an ovarian cancer mouse model
}

\author{
Travis W. Sawyer ${ }^{\mathrm{a}}$, Faith S. Rice ${ }^{\mathrm{b}}$, Jennifer W. Koevary ${ }^{\mathrm{b}}$, Denise C. Connollyc, Kathy Q. Caic, \\ and Jennifer K. Barton ${ }^{\mathrm{a}, \mathrm{b}}$ \\ ${ }^{a}$ University of Arizona, College of Optical Sciences, 1630 E. University Blvd, Tucson, AZ \\ ${ }^{b}$ University of Arizona, Biomedical Engineering, 1127 James E. Rogers Way, Tucson, AZ \\ ${ }^{\mathrm{c}}$ Fox Chase Cancer Center, 333 Cottman Ave, Philadelphia
}

\begin{abstract}
Ovarian cancer is the deadliest gynecologic cancer due to predominantly late diagnosis. Early detection of ovarian cancer can increase 5-year survival rates from $40 \%$ up to $92 \%$, yet no reliable early detection techniques exist. Multiphoton microscopy (MPM) is a relatively new imaging technique with tremendous potential for clinical diagnosis. A sub-modality of MPM is second harmonic generation (SHG) imaging, which generates contrast from anisotropic structures like collagen molecules, enabling the acquisition of detailed molecular structure maps. As collagen is known to change throughout the progression of cancer, MPM is a promising candidate for ovarian cancer screening.

While MPM has shown favorable results in a research environment, it has not yet found broad success in a clinical setting. One major obstacle is the quantitative analysis of the image content. Recently, the application of texture analysis to MPM images has shown success for characterizing the collagen content of the tissue, making it a prime candidate for disease screening. Unfortunately, existing work is limited in its application to ovarian tissue and few texture analysis approaches have been evaluated in this context.

To address these challenges, we applied texture analysis to second harmonic generation (SHG) and two-photon excited fluorescence (TPEF) images of a mouse model (TgMISIIR-TAg) of ovarian cancer. Using features from the grey-level co-occurrence matrix, we find that texture analysis of TPEF images of the ovary can differentiate between genotype with high statistical significance ( $\left.\mathrm{p}_{\mathbf{i}} 0.001\right)$, whereas TPEF and SHG images of the oviducts are most sensitive to age, and SHG images of the ovaries are most sensitive to reproductive status. While these results suggest that texture analysis is suitable for characterizing ovarian tissue health, further work is focused on developing a classification algorithm based on these features, and also to couple the results with a histopathological analysis.
\end{abstract}

Keywords: second harmonic generation, multiphoton imaging, ovarian cancer, texture analysis

\section{INTRODUCTION}

\subsection{Burden of Ovarian Cancer}

Despite concerted efforts to improve patient outcomes, ovarian cancer remains the deadliest gynecologic malignancy in the United States. While ovarian cancer is not exceedingly common, the disease maintains a high mortality rate, with median five-year survival less than $45 \% .^{1}$ One cause of this is the fact that ovarian cancer can grow to a large size before causing signs or symptoms, leading to a high proportion of advanced disease at the time of detection. In fact, a large majority of patients have already experienced spread of their disease to local or distant tissues at initial diagnosis, resulting in a significantly poorer prognosis. ${ }^{2}$

This insidious pattern of disease progression has led to strong interest in the area of ovarian cancer screening, with the ultimate goal of identifying early stage tumors, while the patient is still asymptomatic, allowing more effective treatment. Various screening modalities have been investigated to reduce the burden of the disease

Further author information: (Send correspondence to J.K.B.)

J.K.B.: E-mail: barton@email.arizona.edu, Telephone: 15206214116

Diseases in the Breast and Reproductive System V, edited by Melissa C. Skala, Paul J. Campagnola, Darren M. Roblyer, Proc. of SPIE Vol. 10856, $1085605 \cdot$ C 2019 SPIE · CCC code: 1605-7422/19/\$18 · doi: 10.1117/12.2505825 
including physical examination, transvaginal ultrasound (TVUS), and serum tumor marker measurement (most commonly Ca-125). ${ }^{3}$ Other screening tests and multimodal protocols have also been investigated; however, at this time no routine screening is recommended in average-risk patients. ${ }^{4}$ As such, there remains a strong need for a high-quality, minimally invasive modality for effective detection of early-stage ovarian malignancies.

\subsection{Multiphoton Imaging}

Several different optical imaging techniques have been used to probe ovarian tissue health including fluorescence spectroscopy ${ }^{5}$ multispectral imaging, ${ }^{6}$ confocal imaging, ${ }^{7}$ photoacoustic imaging, ${ }^{8}$ and optical coherence tomography. ${ }^{9}$ One technique that has shown promise in the identification of abnormal ovarian tissue is multiphoton microscopy (MPM). ${ }^{10,11}$ MPM is based on the molecules in tissue interacting with more than one photon simultaneously, allowing for signal generation at a higher energy than the illuminating light. One type of MPM is second-harmonic generation (SHG), in which contrast is formed by the nonlinear scattering by molecules with non-centrosymmetric structure. ${ }^{12}$ The nonlinear nature of the scattering in SHG results in emission light with precisely twice the frequency of the excitation light. Another form of MPM is two-photon excited fluorescence (TPEF), in which an endogenous fluorophore absorbs two photons simultaneously to generate fluorescence emission at a higher frequency. Due to vibrational relaxations and other losses, the frequency of the fluorescent light undergoes a Stokes shift to slightly less than twice the illumination frequency. TPEF allows for utilization of endogenous fluorescence, eliminating the possibility of toxicity or other side effects from exogenous contrast agents, and simplifying the imaging procedure. Endogenous fluorophores in the body include proteins containing aromatic amino acids (tryptophan, tyrosine, phenylalanine), metabolic co-factors such as NADH and FAD, structural proteins such as collagen and elastin, and a variety of other molecules including vitamins and lipopigments. ${ }^{13,14}$

For both SHG and TPEF, three-dimensional images are formed by scanning the focused excitation beam across the tissue. The probability of two-photon interaction scales with the square of the irradiance. ${ }^{15}$ Due to the irradiance of the beam sharply decreasing with distance from the beam focus, this allows for probing a thin section of the sample, without exciting regions of the sample outside the focal plane. ${ }^{16}$ The necessity of high numerical aperture to generate efficient two-photon processes emission yields lateral resolutions of less than 1 $\mu m .{ }^{11}$ TPEF and SHG have the advantage of high resolution, depth resolved imaging, with an imaging depth greater than confocal imaging. The increased depth of imaging is achieved by using near infrared short-pulsed light. The longer wavelengths penetrate deeper than ultraviolet or visible light, which are typically used in single photon techniques such as confocal microscopy and surface fluorescence imaging. Additionally, at near-infrared wavelengths there is minimal absorption and tissue damage.

\subsection{Texture Analysis}

MPM generates contrast that shows changes in endogenous cellular fluorescence (TPEF) and collagen structure (SHG) as a result of ovarian cancer. ${ }^{17-19}$ For example, SHG has been used to show that normal ovaries have thin collagen fibers organized in a net-like structure, whereas malignant ovaries have a denser, wavy collagen structure, which may result from recruitment of activated fibroblasts to the outer rim of the tumor. ${ }^{17,19-21}$ Therefore, analysis of MPM images is focused on quantitatively assessing these structural differences of the tissue to evaluate tissue health.

As MPM provides abundant information about the tissue structure, one of the most promising approaches to quantitation is image texture analysis. Texture analysis in image processing is a general method to describe the local variations in image brightness. Often used in conjunction with the concept of tone, which is related to the varying levels of image brightness, texture characterizes the spatial distribution of the tones in an image. ${ }^{22} \mathrm{~A}$ number of techniques have been developed for texture analysis of images, which can be generally categorized into three groups: statistical, spectral, and structural methods. Statistical methods are based on analyzing image histograms by computing their statistical moments and other properties. ${ }^{23}$ These approaches are best suited to characterize features such as inhomogeneity and contrast. Spectral methods apply autocorrelation and Fourier analysis to evaluate periodic features of an image. Finally, structural approaches decompose the image into a set of sub-patterns, arranged according to certain placement rules. 
Several different approaches have been applied for quantitative analysis of MPM images. One of the most direct is to threshold the images based on brightness in order to evaluate fibrosity of the collagen network. ${ }^{24,25}$ Another common technique is to employ frequency analysis, which quantifies the global spatial frequency content of an image. ${ }^{20,26,27}$ Recently, techniques focusing on local image features have been applied for tissue health classification. These include wavelet ${ }^{28,29}$ and curvelet analysis, ${ }^{30}$ and also by convolving the images with a set of filter patches at different scales and orientations. ${ }^{29}$

Here, we use MPM to image a mouse model of ovarian cancer in vivo. SHG and TPEF images are collected for both the ovaries and the oviducts (murine equivalent structure of fallopian tubes in human) and features are extracted using texture analysis. The oviducts are of interest, as previous work has shown that oviductal markers are expressed in ovarian tumours in TAG + mice; ${ }^{31}$ additionally, recent work has shown that many ovarian carcinomas in humans originate in the fallopian tubes. ${ }^{32}$ In this manuscript, we first describe the details of the mouse model that spontaneously develops ovarian cancer, as well as the imaging protocol and procedure for conducting texture analysis. Then, we report the results of the texture analysis for both SHG and TPEF images of the ovaries and oviducts, showing that texture features can be used to differentiate experimental groups (age, genotype and treatment) with high statistical significance. In particular, TPEF images of the ovaries have the most significant changes with respect to genotype, which is a proxy for disease. Both SHG and TPEF images show significant textural changes in the oviducts for different ages and SHG images of the ovaries are more sensitive to changes in reproductive status. The results suggest that texture analysis of MPM images may be useful as an aid for ovarian cancer screening.

\section{METHODS}

\subsection{Mouse Model}

For this experiment, we used a transgenic mouse model in which females spontaneously develop bilateral epithelial ovarian cancer. ${ }^{33,34}$ Male TAg + mice were bred to female C57Bl/6 (wild type) mice. This resulted in production of offspring that either harbor the TAg + transgene $(\mathrm{TAg}+)$ or are wild type. Female offspring of both genotypes were injected with vehicle (sesame oil) or 4-Vinylcyclohexene diepoxide (VCD) dissolved in sesame oil at a concentration of $80 \mathrm{mg} / \mathrm{kg}$ for 20 days beginning at post-natal day seven. VCD was used to destroy preantral follicles, resulting in early ovarian failure. VCD has previously been used as a model for menopause. ${ }^{35}$ Mice were imaged at four and eight weeks of age. For brevity, we refer to different groups in figures by abbreviating (age-genotype-treatment). For example, 4WV refers to four weeks of age, wild type treated with VCD and 8TS refers to eight weeks of age, TAg + treated with sesame oil.

With this procedure, we have eight different groups to compare ( $2 \times 2 \times 2$ for age, genotype and treatment). A total of 53 mice were imaged at both time points, yielding 13 samples per group except for wild type sesame oil-treated mice, for which there were 14 samples (Table 1). Each of the two ovaries are analyzed, as well as the oviducts. Within these eight groups we have three distinct class designations: age, genotype and treatment (reproductive status). This poses an interesting challenge for class separation based on image analysis, as we expect the structure of the ovary to change due to each of these three processes. Aging and changing reproductive status are natural biological effects, thus it provides a more realistic challenge to separate based on disease with these confounding factors.

Table 1. Number of mice for each experimental groups imaged. *some images were not of suitable quality for analysis and are excluded

\begin{tabular}{|l|l|l|c|l|}
\hline & \multicolumn{2}{|c|}{ VCD } & \multicolumn{2}{c|}{ Sesame Oil } \\
\hline Age & TAg+ & Wild Type & TAg+ & Wild Type \\
\hline 4 Week & 13 & 13 & $12[13]^{*}$ & 14 \\
\hline 8 Week & $11[13]^{*}$ & 13 & 13 & 14 \\
\hline
\end{tabular}

\subsection{Multiphoton Imaging}

Imaging was performed with a single-beam multiphoton microscope (TrimScope, LaVision BioTec, Bielefeld, Germany) using a Titanium:Sapphire laser light source (Chameleon Ultra2, Coherent, UK) that was coupled to the scanner unit, with a pulse width of 120 femtoseconds at the sample. The laser intensity was adjusted 
to $35 \mathrm{~mW}$ average power with an electro-optical modulator (EOM 350-80; Conoptics, Danbury, CT). A waterimmersion, 20X magnification, 0.95-NA objective (Olympus, Center Valley, PA) was used for imaging. The excitation wavelength was set to $780 \mathrm{~nm}$, and SHG and TPEF image data were recorded simultaneously. A bandpass filter FF01-377/50 (Semrock) and a dichroic mirror Di01-R405-25X36 (Chroma) were used to collect light from SHG and a bandpass filter HQ450/100M-2p-25 (Chroma, Bellows Falls, VT) and a dichroic mirror (505dcxr, Chroma) were used to collect light from TPEF. Images were taken at $5 \mu \mathrm{m}$ depth increments from the surface of the tissue to $50-100 \mu \mathrm{m}$ depth. Imaging was completed in less than five minutes per specimen. All images had a $400 \mu m \times 400 \mu m$ field of view and contained $1024 \times 1024$ pixels with 14-bit gray scale resolution.

\subsection{Image Processing}

\subsubsection{Texture Analysis}

We apply two methods of texture analysis to extract features from the acquired images. First is based on constructing and analyzing the Grey-level co-occurrence matrix (GLCM). ${ }^{22}$ The GLCM is a spatial histogram that describes the distribution of grey-level values in an image. Each entry in the GLCM, $p(i, j \mid d, \theta)$, corresponds to the probability of a pixel with a grey-level of $(i)$ being a distance $(d)$ pixels away from a neighboring pixel with a grey-level of $(j)$ in the $(\theta)$ direction (Figure 1a). With an image quantized into $N_{g}$ grey levels, the GLCM is an $N_{g} \times N_{g}$ matrix. For a two-dimensional image, four directions for $(\theta)$ are possible: 0 degree, 45 degree, 90 degree, 135 degree (Figure 1b). In this study, we fix $(d)$ at one pixel, and compute the GLCM for the four possible directions.

(a)

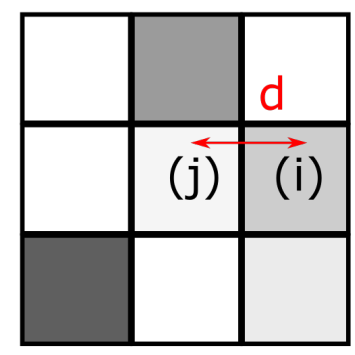

(b)

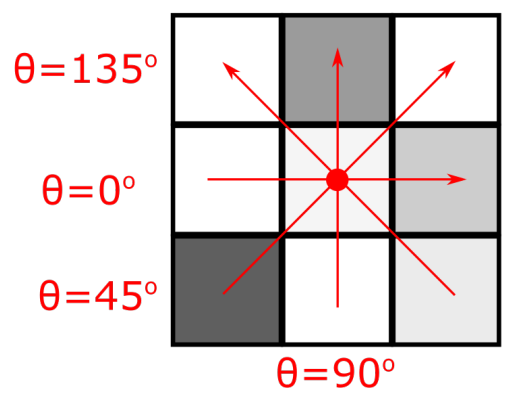

(c)

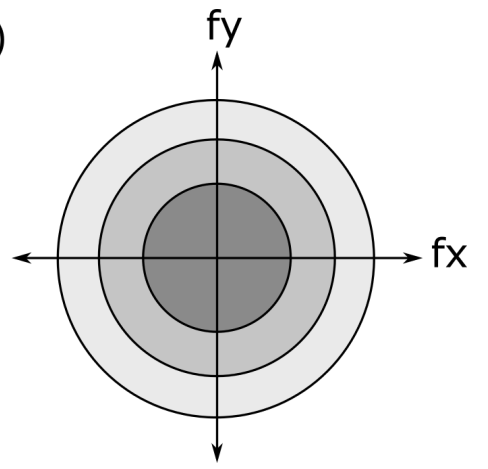

Figure 1. The GLCM is constructed by measuring the probability of two pixel values $\mathrm{i}$ and $\mathrm{j}$ occurring a distance $\mathrm{d}$ from one another (a). This can be done for four different directions in $2 \mathrm{D}$ (b). The texture is also quantified by assessing the frequency distribution by integrating the fractional energy in a disk centered at the origin of coordinates for the Fourier Transform (c).

From the GLCM, we compute thirteen texture features originally proposed by Haralick in $1973^{22}$ to describe the texture of an image as follows:

$$
\begin{aligned}
& \text { Angular Second Moment }=\sum_{i} \sum_{j} p(i, j)^{2}, \\
& \text { Contrast }=\sum_{n=0}^{N_{g}-1} n^{2} \sum_{i=1}^{N_{g}} \sum_{j=1}^{N_{g}} p(i, j) ; \quad n=|i-j|, \\
& \text { Correlation }=\sum_{i} \sum_{j} \frac{(i j) p(i, j)-\mu_{x} \mu_{y}}{\sigma_{x} \sigma_{y}}
\end{aligned}
$$


where $\mu_{x}, \mu_{y}, \sigma_{x}, \sigma_{y}$ are the mean and standard deviations of $p_{x}$ and $p_{y}$, the marginal probability density functions;

$$
\begin{gathered}
\text { Sum of Squares: Variance }=\sum_{i} \sum_{j}(i-\mu)^{2} p(i, j), \\
\text { Inverse Difference Moment }=\sum_{i} \sum_{j} \frac{1}{1+(i-j)^{2}} p(i, j), \\
\qquad \text { Sum Average }=\sum_{i=2}^{2 N_{g}} i p_{x+y}(i),
\end{gathered}
$$

where $x$ and $y$ are the row and column indices of the GLCM and $p_{x+y}(i)$ is the probability of the two indices summing to $x+y$;

$$
\begin{aligned}
& \text { Sum Entropy }=f_{s}=-\sum_{i=2}^{2 N_{g}} p_{x+y}(i) \log \left(p_{x+y}(i)\right), \\
& \text { Sum Variance }=\sum_{i=2}^{2 N_{g}}\left(i-f_{s}\right)^{2} p_{x+y}(i), \\
& \text { Entropy }=-\sum_{i} \sum_{j} p(i, j) \log (p(i, j)), \\
& \text { Difference Variance }=\sum_{i=0}^{N_{g}-1} i^{2} p_{x-y}(i),
\end{aligned}
$$

Difference Entropy $=-\sum_{i} \sum_{j} p_{x-y}(i) \log \left(p_{x-y}(i)\right)$,

$$
\text { Info. Measure of Correlation } 1=\frac{S_{x y}-S_{x y 1}}{\max \left(S_{x}, S_{y}\right)} \text {, }
$$

$$
\text { Info. Measure of Correlation } 2=\left(1-\exp \left(-2\left(S_{x y 2}-S_{x y}\right)\right)\right)^{1 / 2}
$$

We compute the features shown in Eqs. (1) (13) for each GLCM corresponding to the four possible 2D directions for $(\theta)$. For a stack of SHG or TPEF images, the image with the highest signal is used for analysis. We then average the features for the four directions to produce a single set of features; by averaging over 
the different directions, we effectively create rotationally-invariant features, which is important when imaging spatially unconstrained objects such as biological tissue.

These texture features can be interpreted to understand what properties of the image are quantified by each. For example, here we describe five of the most common features used to describe image texture. The Angular Second Moment, also known as energy (1), is found by summing the squared values in the GLCM; therefore, this feature yields a high value when the image is homogeneous; in other words: the GLCM has a few, large entries. In contrast, the entropy (9), introduced by ${ }^{36}$ describes the inhomogeneity of the image. By multiplying the GLCM entries by their logarithm, smaller values are amplified, thus having the opposite effect of the Energy feature. Both the contrast and variance (2 and 4 ) are well-known statistical parameters that measures the variations in tone by providing higher weight to GLCM entries that are far from the diagonal, which correspond to areas of high contrast. Essentially the opposite of variance, the inverse-difference moment amplifies the diagonal entries of the GLCM. Physically, this corresponds to a high feature value for low-contrast areas, such as large patches of uniform grey-level. Each of the other Haralick features provides additional information regarding image texture, and are described in. ${ }^{22}$

\subsubsection{Frequency Analysis}

We compute a second set of features by analyzing the magnitude of the discrete Fast Fourier transform (FFT) of the image in 2D. The FFT of the image data is calculated and stored in a matrix where the central value in the matrix represents the DC frequency component. We then integrate the magnitude of the spatial frequencies in a disk centered at the origin (Figure 1c). The value is normalized to the total signal magnitude, representing the percentage of the total signal contained in a given disk. The radius of this disk is increased iteratively and the total signal proportion is recorded until the radius has reached $80 \%$ of the maximum, beyond which only noise remains. This is effectively the cumulative distribution function (CDF) of the energy density; taking the difference between the values for any two radii gives the proportion of energy contained within a specific frequency band. Images that are highly homogenous would have higher energy density for associated with lower spatial frequencies. On the other hand, images with more inhomogeneity would have more energy density corresponding to higher spatial frequency. We then parameterize the distribution by fitting the CDF curve to the following equation:

$$
y=\alpha x^{\beta}+\gamma
$$

Where $y$ is the value of the CDF for a given spatial frequency $x$. The frequency distribution is thus described by the three features: $\alpha, \beta$, and $\gamma$, which are used to differentiate between the different experimental groups. The analyses were completed in Python using a computer with an Intel Core I-4710HQ CPU (2.50 GHz) and 16 GB DDR3L memory.

\subsection{Statistics}

Statistical analysis for the texture analysis for SHG and TPEF images was completed using a linear mixed effects model with random intercepts in order to account for within-subject and three-way interactions between age, genotype and treatment. In addition, Tukey-Kramer p-value adjustment was done to account for multiple comparisons. Differences are considered statistically significant for $\mathrm{p}<0.05$ (denoted ${ }^{*}$ ), $\mathrm{p}<0.01$ (denoted ${ }^{* *}$ ), and $\mathrm{p}<0.001$ (denoted ${ }^{* *}$ ).

\section{RESULTS AND DISCUSSION}

\subsection{Multiphoton Imaging}

Representative SHG and TPEF images for the oviducts and ovaries are shown in Figure 2 for a health mouse at eight weeks, and in Figure 3 for a diseased TAg+ mouse at 8 weeks, where histopathology indicates that $50 \%$ of the mouse ovaries and oviducts had malignancy. In particular, the structure of the ovaries for both SHG and TPEF is visibly more irregular (subfigures $\mathrm{c}$ and d). Furthermore, the collagen structure of the oviducts appears more disordered in the TAg + mouse compared to the wild type. While the TPEF image of the oviducts does 
not seem particularly different in a visual inspection, this may be due to the particular field of view, as only $50 \%$ of the organ was determined to be diseased by histopathology. While the images seem to have qualitative differences, this does not necessarily represent the entire sample and thus has no statistical power. Therefore, it is necessary to conduct a rigorous analysis of the image texture, as is done is the following section.
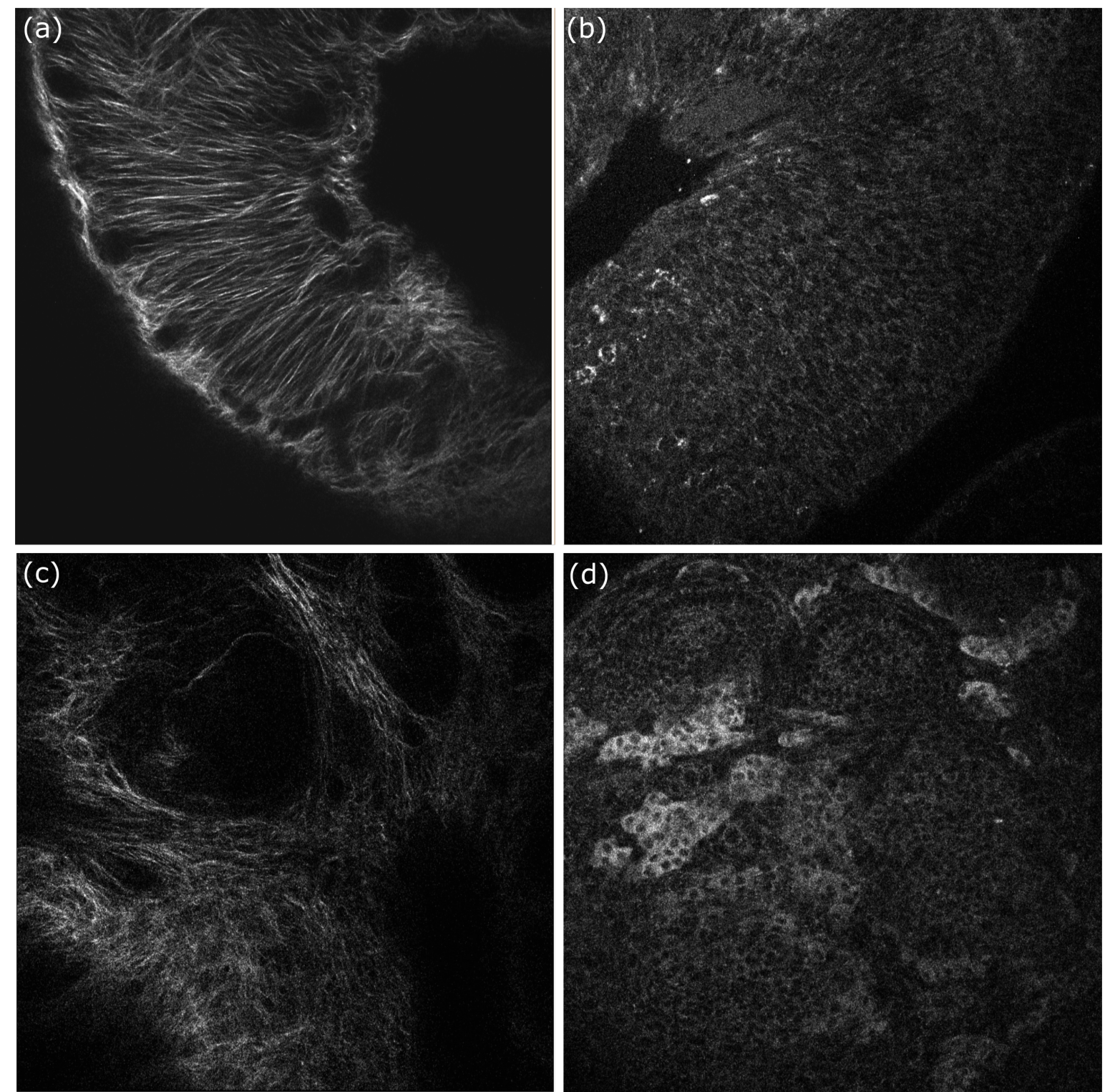

Figure 2. Example images taken from a healthy specimen. SHG image of oviduct (a), TPEF image of oviduct (b), SHG image of ovary (c), TPEF image of ovary (d).

\subsection{Texture Analysis}

Selected results for features from the GLCM analysis are shown in Figure 4. Using the features to perform pairwise comparison tests between different mouse groups, multiple features are significant using all four imaging situations: SHG and TPEF for both the ovaries and oviducts. Given the large number of features and comparisons, we select a subset for display. In the following section, however, we summarize the results comprehensively for three aggregated comparisons between age, genotype and treatment. In general, we find that the most challenging group to differentiate is genotype; however, the most sensitive features are correlation-based features of 

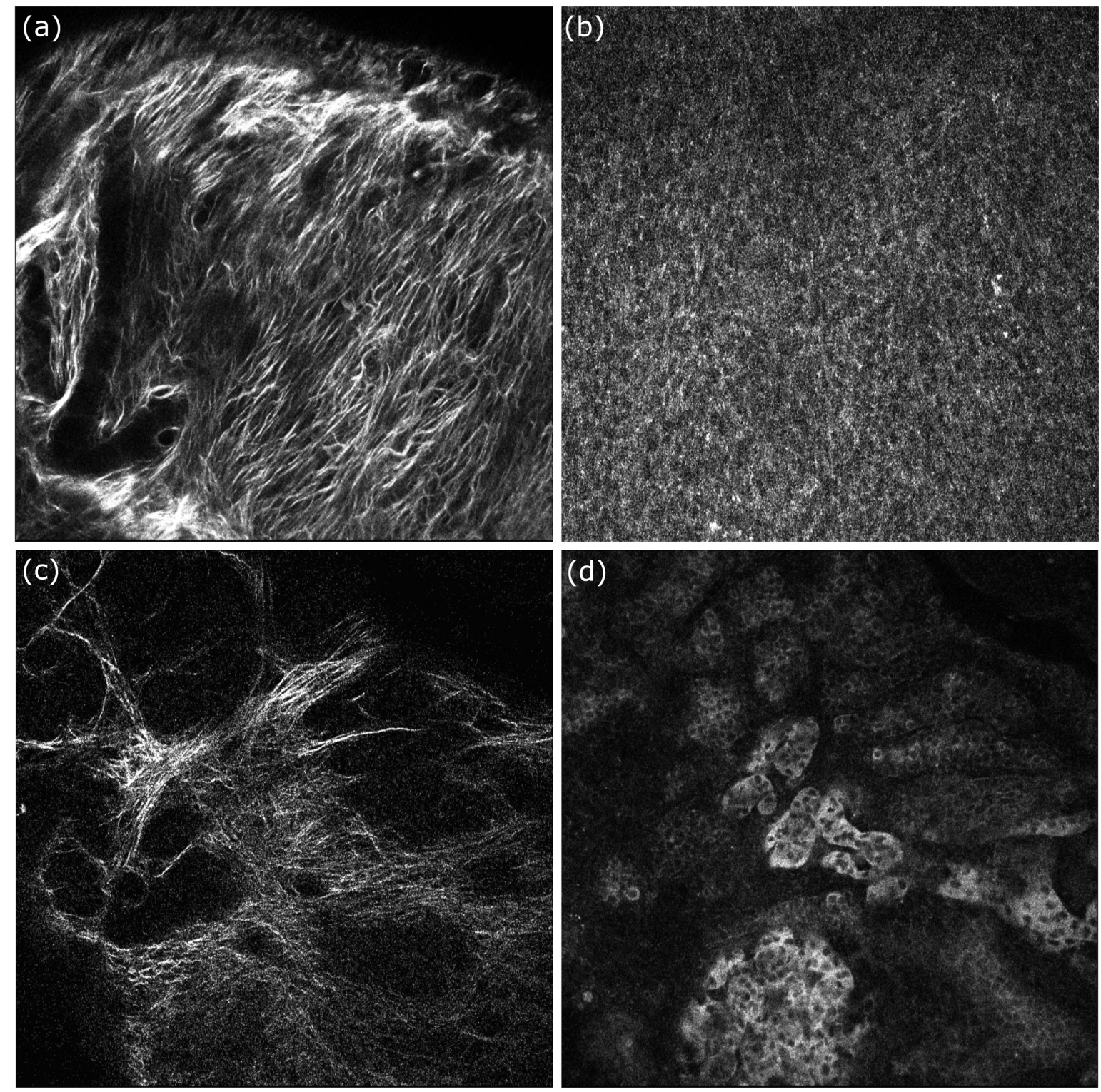

Figure 3. Example images taken from a specimen with ovarian cancer. SHG image of oviduct (a), TPEF image of oviduct (b), SHG image of ovary (c), TPEF image of ovary (d). Histopathology indicates over $50 \%$ of the ovary was diseased tissue. 
SHG and TPEF imaging of the ovaries (Figure 4a, b). However, we also find that other complimentary features such as variance and entropy based features for SHG and TPEF imaging of the oviducts (Figure 4c, d) provide high statistical significance for separating groups. In general, age tends to yield the highest differences between features, indicating that this may be a particularly challenging effect to overcome. It is important to note as well, that as cancer will develop as the mouse ages, the differences between genotype might become more apparent with 8 week mice.
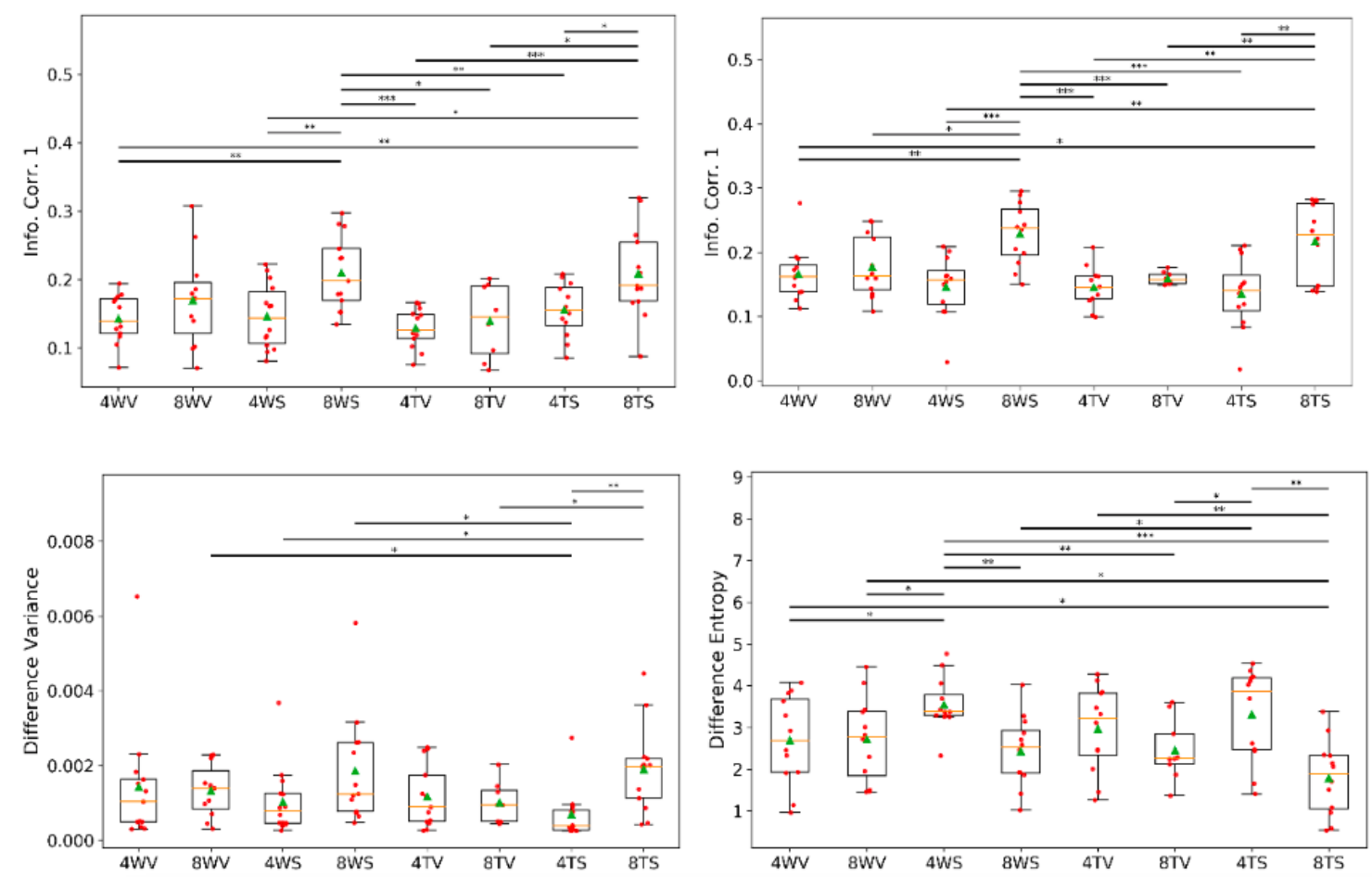

Figure 4. GLCM texture features for (a) SHG Ovaries, (b) SHG FT, (c) TPEF Ovaries, (d) TPEF FT.

While it is interesting to inspect the statistical variations between individual experimental groups, it is more telling to evaluate whether the given feature is sensitive to changes for a given parameter in aggregate (e.g, changes in genotype across all groups). We do this in the following section using a linear mixed effects model with random intercepts in order to account for within-subject and three-way interactions between age, genotype and treatment.

\subsection{Variations by Group}

The aggregated differences induced by age, genotype and treatment are shown in Tables 2-4, respectively. The second of these has the most diagnostic relevance, as analysis of HE and TAg-stained tissue sections indicates that all TAg+ mice have TAg + tumor cells in their ovaries by 8 week of age. In this case, we see that TPEF imaging of the ovaries produces seven features that have high statistical significance for differentiating between genotype. The confounding effects of age and treatment may reduce the fidelity of the feature discrimination for genotype. However, this is a more realistic assessment, as cancer can develop at any age and reproductive status. SHG imaging shows no significant features for differentiating genotype; however, several features are sensitive to both treatment and age

These results are encouraging; however, several challenges remain before bringing the approach toward practical implementation as a physician aid. First, while the mouse model used here is an interesting and unique classification problem, the classification of tissue health is the ultimate goal for such a tool. Here, the mouse genotype is used as a proxy for disease, since the $\mathrm{TAg}+$ genotype is used to induce ovarian cancer. Considering this, the results for genotype are of particular interest, were we see that TPEF imaging of the ovaries provides 

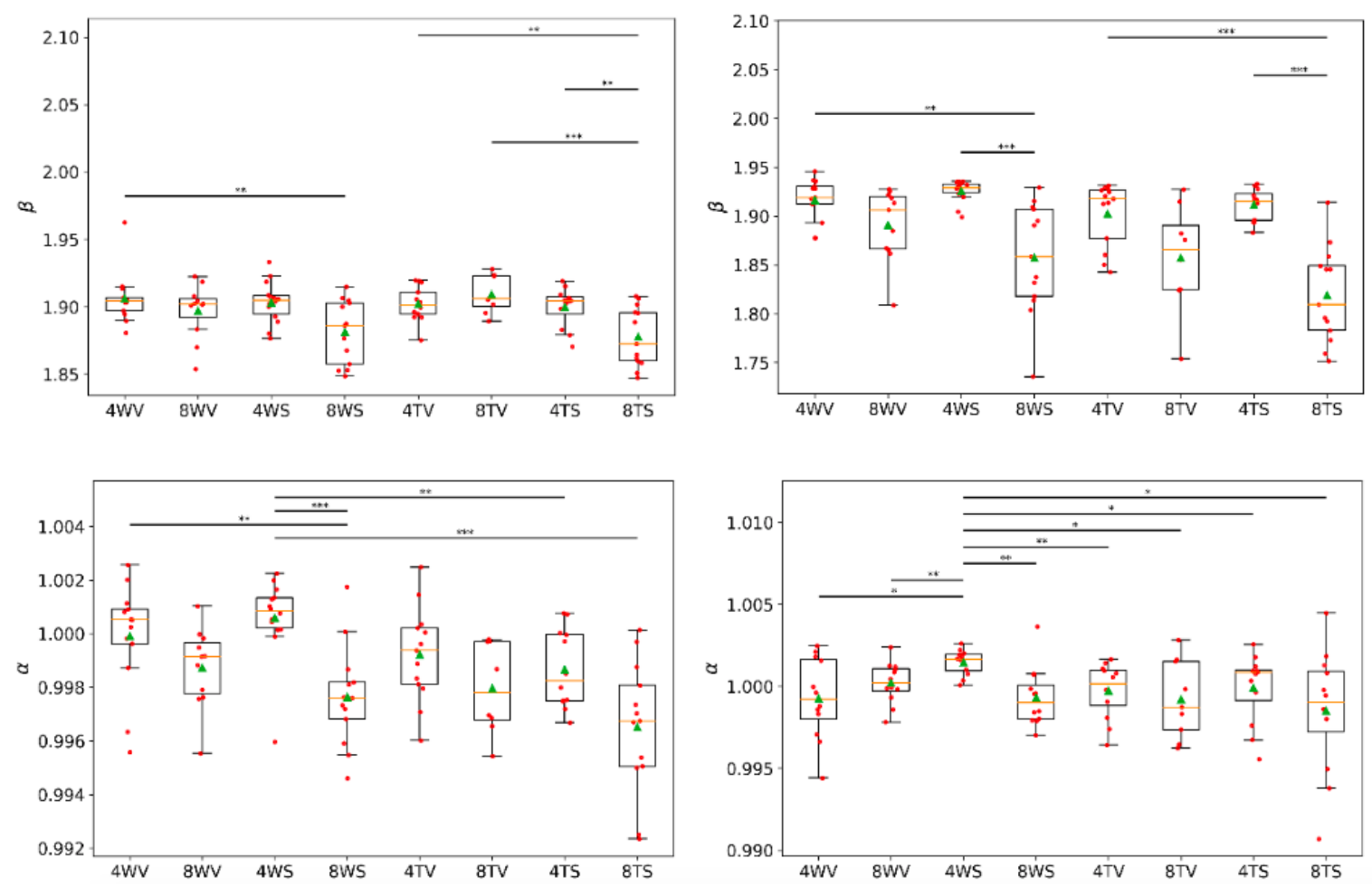

Figure 5. Frequency-based texture features for (a) SHG Ovaries, (b) SHG FT, (c) TPEF Ovaries, (d) TPEF FT.

\begin{tabular}{|l|c|c|c|c|}
\hline & \multicolumn{2}{|c|}{ Ovaries } & \multicolumn{2}{c|}{ oviducts } \\
\hline Feature & SHG & TPEF & SHG & TPEF \\
\hline Angular Second Moment & - & $*$ & $* *$ & - \\
\hline Contrast & - & $*$ & - & $* * *$ \\
\hline Correlation & - & $* * *$ & $* * *$ & $*$ \\
\hline Sum of Squares: Variance & - & - & - & $* *$ \\
\hline Inverse Difference Moment & - & $* * *$ & - & $* *$ \\
\hline Sum Average & - & - & - & $* *$ \\
\hline Sum Entropy & - & $*$ & - & $*$ \\
\hline Sum Variance & - & $*$ & $*$ & $* *$ \\
\hline Entropy & - & $*$ & - & $* *$ \\
\hline Difference Variance & - & $*$ & - & $*$ \\
\hline Difference Entropy & - & $* * *$ & - & $* * *$ \\
\hline Info. Measure of Correlation 1 & $*$ & $* * *$ & $* * *$ & $* * *$ \\
\hline Info. Measure of Correlation 2 & - & $* * *$ & $* * *$ & - \\
\hline$\alpha$ & - & $* * *$ & - & - \\
\hline$\beta$ & $* * *$ & $* * *$ & $* * *$ & $* *$ \\
\hline$\gamma$ & $* * *$ & - & - & - \\
\hline
\end{tabular}

Table 2. Significance of features for each imaging modality and organ for differentiating mice by age. 


\begin{tabular}{|l|c|c|c|c|}
\hline & \multicolumn{2}{|c|}{ Ovaries } & \multicolumn{2}{c|}{ oviducts } \\
\hline Feature & SHG & TPEF & SHG & TPEF \\
\hline Angular Second Moment & - & - & - & - \\
\hline Contrast & - & - & - & - \\
\hline Correlation & - & $* * *$ & - & $*$ \\
\hline Sum of Squares: Variance & - & $* *$ & - & - \\
\hline Inverse Difference Moment & - & - & - & - \\
\hline Sum Average & - & $*$ & - & - \\
\hline Sum Entropy & - & $*$ & - & - \\
\hline Sum Variance & - & - & - & - \\
\hline Entropy & - & - & - & - \\
\hline Difference Variance & - & - & - & - \\
\hline Difference Entropy & - & - & - & - \\
\hline Info. Measure of Correlation 1 & - & $*$ & - & - \\
\hline Info. Measure of Correlation 2 & - & $*$ & - & - \\
\hline$\alpha$ & - & $* *$ & - & - \\
\hline$\beta$ & - & $* *$ & - & - \\
\hline$\gamma$ & - & - & - & - \\
\hline
\end{tabular}

Table 3. Significance of features for each imaging modality and organ for differentiating mice by treatment.

\begin{tabular}{|l|c|c|c|c|}
\hline & \multicolumn{2}{|c|}{ Ovaries } & \multicolumn{2}{c|}{ oviducts } \\
\hline Feature & SHG & TPEF & SHG & TPEF \\
\hline Angular Second Moment & - & - & - & - \\
\hline Contrast & - & - & - & - \\
\hline Correlation & $* *$ & $*$ & - & $*$ \\
\hline Sum of Squares: Variance & - & - & - & - \\
\hline Inverse Difference Moment & - & - & - & - \\
\hline Sum Average & - & - & - & - \\
\hline Sum Entropy & - & - & - & - \\
\hline Sum Variance & - & - & - & - \\
\hline Entropy & - & - & - & - \\
\hline Difference Variance & - & - & - & - \\
\hline Difference Entropy & - & - & - & - \\
\hline Info. Measure of Correlation 1 & $* *$ & $*$ & - & - \\
\hline Info. Measure of Correlation 2 & $* *$ & $*$ & - & - \\
\hline$\alpha$ & - & - & - & - \\
\hline$\beta$ & $* *$ & - & $*$ & - \\
\hline$\gamma$ & - & - & - & - \\
\hline
\end{tabular}

Table 4. Significance of features for each imaging modality and organ for differentiating mice by genotype. 
the highest fidelity. Even so, conducting an analysis with histopathology results is of particular interest to truly determine whether the differences shown correlate to cancer.

Furthermore, the results we report are descriptive statistics. While useful to observe the variations of each feature, the next step is to implement a classification algorithm trained using these features to try and differentiate between tissue types. This will ultimately show whether texture analysis of MPM images has high potential as a diagnostic aid. Our previous work has shown that using linear discriminant analysis is a useful approach to classification for OCT imaging of the ovaries. ${ }^{37}$ We currently are working to implement a similar scheme using these imaging modalities; other possibilities for classification algorithms include machine learning approaches. Finally, while the results indicate that MPM is effective for assessing ovarian tissue health, it is limited by a small field of view and is sensitive to particular forms of endogenous contrast. Therefore, it could be more effective to couple MPM with another complimentary imaging modality such as wide-field fluorescence imaging (with exogenous contrasts) or OCT.

\section{CONCLUSIONS}

In this manuscript, we assess the potential of multiphoton microscopy for evaluating ovarian tissue health. We image a transgenic mouse model that spontaneously develops ovarian cancer in vivo using both second harmonic generation and two-photon excited fluorescence imaging. By using texture analysis based on the grey-level co-occurrence matrix, as well as features describing the frequency content of these images, we show that it is possible to differentiate between experimental groups (age, genotype and reproductive status) with high statistical significance $(\mathrm{p}<0.001)$. While these results are promising, next steps include developing a classification scheme using methods such as linear discriminant analysis and machine learning. Furthermore, the TAg genotype is used as a proxy for disease. While preliminary histology shows that all TAg mice developed disease by 8 weeks, conducting a rigorous analysis with histopathology results is of particular interest to truly determine whether the differences shown correlate to cancer.

\section{REFERENCES}

[1] Barnholtz-Sloan, J. S., Schwartz, A. G., Qureshi, F., Jacques, S., Malone, J., and Munkarah, A. R., "Ovarian cancer: Changes in patterns at diagnosis and relative survival over the last three decades," Am. J. Obstet. Gynecol. 189(4), 1120-1127 (2003).

[2] Maringe, C., Walters, S., Butler, J., Coleman, M. P., Hacker, N., Hanna, L., Mosgaard, B. J., Nordin, A., Rosen, B., Engholm, G., Gjerstorff, M. L., Hatcher, J., Johannesen, T. B., McGahan, C. E., Meechan, D., Middleton, R., Tracey, E., Turner, D., Richards, M. A., and Rachet, B., "Stage at diagnosis and ovarian cancer survival: Evidence from the international cancer benchmarking partnership," Gynecol. Oncol. 127(1), 75-82 (2012).

[3] Carlson, K. J., "Screening for ovarian cancer," UpToDate (2017).

[4] Moyer, V. A., "Screening for ovarian cancer: U.S. Preventive services task force reaffirmation recommendation statement," (2012).

[5] George, R., Chandrasekaran, A., Brewer, M. a., Hatch, K. D., and Utzinger, U., "Clinical research device for ovarian cancer detection by optical spectroscopy in the ultraviolet C-visible.," Journal of biomedical optics (2010).

[6] Van Dam, G. M., Themelis, G., Crane, L. M., Harlaar, N. J., Pleijhuis, R. G., Kelder, W., Sarantopoulos, A., De Jong, J. S., Arts, H. J., Van Der Zee, A. G., Bart, J., Low, P. S., and Ntziachristos, V., "Intraoperative tumor-specific fluorescence imaging in ovarian cancer by folate receptor- $\alpha$ targeting: First in-human results," Nature Medicine (2011).

[7] Tanbakuchi, A. A., Udovich, J. A., Rouse, A. R., Hatch, K. D., and Gmitro, A. F., "In vivo imaging of ovarian tissue using a novel confocal microlaparoscope," American Journal of Obstetrics and Gynecology (2010).

[8] Wang, T., Yang, Y., Alqasemi, U., Kumavor, P. D., Wang, X., Sanders, M., Brewer, M., and Zhu, Q., "Characterization of ovarian tissue based on quantitative analysis of photoacoustic microscopy images," Biomedical Optics Express (2013). 
[9] Huang, D., Swanson, E., Lin, C., Schuman, J., Stinson, W., Chang, W., Hee, M., Flotte, T., Gregory, K., Puliafito, C., and et, a., "Optical coherence tomography," Science 254(5035), 1178-1181 (1991).

[10] Watson, J. M., Marion, S. L., Rice, P. F., Utzinger, U., Brewer, M. A., Hoyer, P. B., and Barton, J. K., "Two-Photon Excited Fluorescence Imaging of Endogenous Contrast in a Mouse Model of Ovarian Cancer," (3 2013).

[11] Welge, W. A., DeMarco, A. T., Watson, J. M., Rice, P. S., Barton, J. K., and Kupinski, M. A., "Diagnostic potential of multimodal imaging of ovarian tissue using optical coherence tomography and second-harmonic generation microscopy," J. Med. Imaging 1 (2014).

[12] Campagnola, P. J. and Loew, L. M., "Second-harmonic imaging microscopy for visualizing biomolecular arrays in cells, tissues and organisms," (2003).

[13] Richards-Kortum, R. and Sevick-Muraca, E., "QUANTITATIVE OPTICAL SPECTROSCOPY FOR TISSUE DIAGNOSIS," Annual Review of Physical Chemistry 47, 555-606 (10 1996).

[14] Lakowicz, J. R., [Principles of Fluorescence Spectroscopy] (2006).

[15] Denk, W., Strickler, J. H., and Webb, W. W., "Two-photon laser scanning fluorescence microscopy.," Science (1990).

[16] Ustione, A. and Piston, D. W., "A simple introduction to multiphoton microscopy," Journal of Microscopy (2011).

[17] Kirkpatrick, N. D., Brewer, M. A., and Utzinger, U., "Endogenous optical biomarkers of ovarian cancer evaluated with multiphoton microscopy," Cancer Epidemiology Biomarkers and Prevention (2007).

[18] Williams, R. M., Flesken-Nikitin, A., Ellenson, L. H., Connolly, D. C., Hamilton, T. C., Nikitin, A. Y., and Zipfel, W. R., "Strategies for High Resolution Imaging of Epithelial Ovarian Cancer by Laparoscopic Nonlinear Microscopy," Translational Oncology (2010).

[19] Nadiarnykh, O., LaComb, R. B., Brewer, M. A., and Campagnola, P. J., "Alterations of the extracellular matrix in ovarian cancer studied by Second Harmonic Generation imaging microscopy," BMC Cancer (2010).

[20] Adur, J., Pelegati, V. B., Costa, L. F. L., Pietro, L., de Thomaz, A. A., Almeida, D. B., Bottcher-Luiz, F., Andrade, L. A. L. A., and Cesar, C. L., "Recognition of serous ovarian tumors in human samples by multimodal nonlinear optical microscopy," Journal of Biomedical Optics (2011).

[21] Granot, D., Addadi, Y., Kalchenko, V., Harmelin, A., Kunz-Schughart, L. A., and Neeman, M., "In vivo imaging of the systemic recruitment of fibroblasts to the angiogenic rim of ovarian carcinoma tumors," Cancer Research (2007).

[22] Haralick, R., Shanmugan, K., and Dinstein, I., "Textural features for image classification," (1973).

[23] Haralick, R. M., "Statistical and structural approaches to texture," Proc. IEEE 67(5), 786-804 (1979).

[24] Strupler, M., Pena, A.-M., Hernest, M., Tharaux, P.-L., Martin, J.-L., Beaurepaire, E., and Schanne-Klein, M.-C., "Second harmonic imaging and scoring of collagen in fibrotic tissues," Optics Express (2007).

[25] Tai, D. C. S., Tan, N., Xu, S., Kang, C. H., Chia, S. M., Cheng, C. L., Wee, A., Wei, C. L., Raja, A. M., Xiao, G., Chang, S., Rajapakse, J. C., So, P. T. C., Tang, H.-H., Chen, C. S., and Yu, H., "Fibro-CIndex: comprehensive, morphology-based quantification of liver fibrosis using second harmonic generation and two-photon microscopy," Journal of Biomedical Optics (2009).

[26] Cicchi, R., Kapsokalyvas, D., De Giorgi, V., Maio, V., Van Wiechen, A., Massi, D., Lotti, T., and Pavone, F. S., "Scoring of collagen organization in healthy and diseased human dermis by multiphoton microscopy," Journal of Biophotonics (2010).

[27] Ambekar, R., Lau, T.-Y., Walsh, M., Bhargava, R., and Toussaint, K. C., "Quantifying collagen structure in breast biopsies using second-harmonic generation imaging," Biomedical Optics Express (2012).

[28] Plotnikov, S., Juneja, V., Isaacson, A. B., Mohler, W. A., and Campagnola, P. J., "Optical clearing for improved contrast in second harmonic generation imaging of skeletal muscle," Biophysical Journal (2006).

[29] Tilbury, K., Hocker, J., Wen, B. L., Sandbo, N., Singh, V., and Campagnola, P. J., "Second harmonic generation microscopy analysis of extracellular matrix changes in human idiopathic pulmonary fibrosis," Journal of Biomedical Optics (2014).

[30] Conklin, M. W., Eickhoff, J. C., Riching, K. M., Pehlke, C. A., Eliceiri, K. W., Provenzano, P. P., Friedl, A., and Keely, P. J., "Aligned collagen is a prognostic signature for survival in human breast carcinoma," American Journal of Pathology (2011). 
[31] Gabbasov, R., Xiao, F., Howe, C. G., Bickel, L. E., O’Brien, S. W., Benrubi, D., Do, T.-V., Zhou, Y., Nicolas, E., Cai, K. Q., Litwin, S., Seo, S., Golemis, E. A., and Connolly, D. C., "NEDD9 promotes oncogenic signaling, a stem/mesenchymal gene signature, and aggressive ovarian cancer growth in mice," Oncogene 37, 4854-4870 (8 2018).

[32] Labidi-Galy, S. I., Papp, E., Hallberg, D., Niknafs, N., Adleff, V., Noe, M., Bhattacharya, R., Novak, M., Jones, S., Phallen, J., Hruban, C. A., Hirsch, M. S., Lin, D. I., Schwartz, L., Maire, C. L., Tille, J. C., Bowden, M., Ayhan, A., Wood, L. D., Scharpf, R. B., Kurman, R., Wang, T. L., Shih, I. M., Karchin, R., Drapkin, R., and Velculescu, V. E., "High grade serous ovarian carcinomas originate in the fallopian tube," Nature Communications (2017).

[33] Connolly, D. C., Bao, R., Nikitin, A. Y., Stephens, K. C., Poole, T. W., Hua, X., Harris, S. S., Vanderhyden, B. C., and Hamilton, T. C., "Female mice chimeric for expression of the simian virus 40 TAg under control of the MISIIR promoter develop epithelial ovarian cancer," Cancer Res. 63(6), 1389-1397 (2003).

[34] Hensley, H., Quinn, B. A., Wolf, R. L., Litwin, S. L., Mabuchi, S., Williams, S. J., Williams, C., Hamilton, T. C., and Connolly, D. C., "Magnetic resonance imaging for detection and determination of tumor volume in a genetically engineered mouse model of ovarian cancer," Cancer Biology and Therapy (2007).

[35] Romero-Aleshire, M. J., Diamond-Stanic, M. K., Hasty, A. H., Hoyer, P. B., and Brooks, H. L., "Loss of ovarian function in the VCD mouse-model of menopause leads to insulin resistance and a rapid progression into the metabolic syndrome.," Am. J. Physiol. Regul. Integr. Comp. Physiol. 297(3), 587-92 (2009).

[36] Shannon, C. E., "A Mathematical Theory of Communication," Bell Syst. Tech. J. 27(3), 379-423 (1948).

[37] Sawyer, T., Chandra, S., Rice, P., Koevary, J., and Barton, J., "Three-dimensional texture analysis of optical coherence tomography images of ovarian tissue.," Phys. Med. Biol. 63(23) (2018). 